\title{
DIFFERENCES IN THE INCIDENCE OF ARMOR BETWEEN INDO-WEST PACIFIC AND WESTERN ATLANTIC/CARIBBEAN MURICINE GASTROPODS: ESCALATION OR PASSIVE EVOLUTION?
}

MILLER, Daniel J., Dept. of the Geophysical Sciences, University of Chicago, 5734 S. Ellis Ave., Chicago, IL 60637, U.S.A.

Previous comparisons of Recent shelled molluscan faunas from the Indo-West Pacific, the East pacific and the tropical western Atlantic/Caribbean have revealed differences in the expression of shell architectural features that are believed to play a role in resisting predators. In general, the Indo-West Pacific fauna displays a higher incidence of anti-predatory architectures than those from the East Pacific and western Atlantic/Caribbean. These differences have been interpreted as evidence that biotic interactions have been more intense in the Indo-West Pacific than in the other biogeographic areas.

Another interpretation of these patterns is possible, however. If the evolution of a prey clade begins at or close to a morphological boundary (e.g., no spines) then subsequent morphological evolution and diversification will be constrained in a direction away from that boundary (i.e., more spines) and will yield character distributions that are asymmetric and "passive" in appearance. If passive evolution occurs in two regions that differ in their evolutionary rates and/or the time available for evolution, then two terminal distributions may be produced that are fundamentally the same but differ in the incidence of extreme morphologies (by virtue of differences in the length of distribution tails). Consequently, it may be possible to generate differences in the incidence and expression of armor via passive processes. While these differences may satisfy, in part, a definition of escalation, the underlying process is very different from the active evolution implicit in the escalation hypothesis.

In this study I document the incidence of anti-predatory architecture in Cenozoic muricine gastropods from the Indo-West Pacific and western Atlantic/Caribbean. Maximum length, number of axial thickenings on the body whorl, and number of spines on the apertural varix were recorded for 108 Indo-West Pacific and 61 western Atlantic/Caribbean muricines. These data were used to test 1) if differences observed in previous studies of other groups are also apparent in muricines and 2) if the differences can be best understood as the result of passive evolution or active escalation.

Analyses of these data show that the incidence of spines is indeed significantly greater in the Indo-West Pacific, as is size. Moreover, a comparison of the distributions revealed that these are not simply similar distributions with different tail lengths but that there are significant differences in the distribution modes as well. The distributions of axial elements in the two regions, however, did not differ significantly.

While these results provide tentative support for an escalation scenario in that the differences can not be explained simply as differences in the length of distribution tails, there is an additional complication. The null hypothesis of passive diffusion used here assumes that diffusion in both regions was initiated at the same point with respect to the morphological boundary. If, however, diffusion started from two different points then the result would be terminal distributions that differ in mode. Thus, it is necessary to explore these patterns in more detail before a passive mechanism is rejected. Current analyses are focussing on comparing distributions for fossil taxa from the two regions as well as applying McShea's subclade test to determine if distributions are passive or driven. 